\title{
Parâmetros cinemáticos da marcha de criança com paralisia cerebral: Comparaçáo entre diferentes formas de apoio ${ }^{2}$
}

\author{
Micheli Martinello ${ }^{a}$, Daiane Lazzeri de Medeiros ${ }^{b}$, Elaine Carmelita Piucco ${ }^{a}$, \\ Lilian Gerdi Kittel Ries ${ }^{b}$

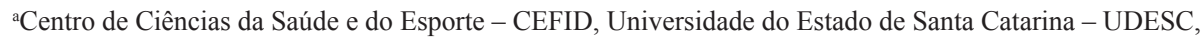 \\ Florianópolis, SC, Brasil \\ 'Programa de Pós-graduação em Fisioterapia, Centro de Ciências da Saúde e do Esporte - CEFID, \\ Universidade do Estado de Santa Catarina - UDESC, Florianópolis, SC, Brasil
}

\begin{abstract}
Resumo: O objetivo deste estudo foi avaliar características cinemáticas da marcha de uma criança com diplegia espástica durante a deambulação auxiliada por diferentes modalidades de apoio (apoio de uma muleta, duas muletas e cintura escapular) e assim determinar com base nas características biomecânicas da marcha a forma mais adequada de treinar a deambulação de uma criança com diplegia espástica. Participou do estudo uma criança de nove anos de idade, do sexo feminino e com diagnóstico de paralisia cerebral do tipo diplegia espástica. Para avaliação clínica da função motora grossa foi utilizado o Gross Motor Function Classification System - GMFCS, o Gross Motor Function Measure - GMFM-88 e a Escala Modificada de Ashworth. A criança realizou cinco repetições da marcha com cada modalidade de apoio e por meio de análise cinemática foram obtidas variáveis espaço-temporais e angulares do quadril e joelho no plano sagital. Os valores que permaneceram mais próximos da marcha no desenvolvimento motor típico foram os obtidos na marcha com duas muletas, seguida do apoio escapular e, por último, da marcha com uma muleta. Verificou-se que a marcha com duas muletas foi a modalidade de apoio mais indicada considerando-se a funcionalidade e independência da criança avaliada. Contudo, a marcha com apoio da cintura escapular pode ser indicada para o treinamento de marcha durante a prática clínica, por estimular diferentes parâmetros espaço-temporais e angulares.
\end{abstract}

Palavras-chave: Marcha, Criança, Paralisia Cerebral.

\section{Kinematic gait parameters of a child with cerebral palsy: Comparison between different forms of support}

\begin{abstract}
The aim of this study was to evaluate the kinematic characteristics of gait in children with spastic diplegia aided by different forms of support (one crutch, two crutches, and scapular girdle), and thus determine the most appropriate way to train gait in children with spastic diplegia based on biomechanical characteristics. The study subject was a female, nine-year-old child, diagnosed with cerebral palsy of spastic diplegia type. The following instruments were used for clinical evaluation of gross motor function: Gross Motor Function Classification System - GMFCS, Gross Motor Function Measure - GMFM 88, and Modified Ashworth Scale. The child performed five repetitions of gait with each type of support. By kinematic analysis, spatiotemporal and angular variables of hip and knee in the sagittal plane were obtained. The values closest to typical gait were obtained with 2 crutches, followed by support with scapular girdle and one crutch. It was possible to observe that gait with two crutches was the most appropriate form of support, considering the functionality and independence of the child assessed; however, gait supported by scapular girdle may be appropriate for gait training in clinical practice, because it stimulates different spatiotemporal and angular parameters.
\end{abstract}

Keywords: Gait, Child, Cerebral Palsy.

Autor para correspondência: Daiane Lazzeri de Medeiros, Centro de Ciências da Saúde e do Esporte, Universidade do Estado de Santa Catarina, Rua Pascoal Simone, 358, Coqueiros, CEP 88080-350, Florianópolis, SC, Brasil, e-mail: daialazzeri@hotmail.com

Recebido em 2/10/2012; Revisão em 25/2/2013; Aceito em 27/5/2013. 


\section{Introdução}

Segundo Berker e Yalçin (2008, p.1209) na Paralisia Cerebral (PC):

[...] a alteração de tônus muscular, déficits no equilíbrio, fraqueza muscular e controle motor seletivo comprometido podem resultar em contraturas e deformidades, as quais podem afetar a rotina diária dessa população (BEKER; YALÇIN, 2008, p. 1209).

Mesmo que o dano cerebral não seja progressivo, a incapacidade decorrente da lesáo pode aumentar ao longo do tempo (RIES; BÉRZIN, 2005). As desordens da postura e do movimento ocasionam atraso do desenvolvimento neuropsicomotor e deformidades musculoesqueléticas. É importante atuar na prevenção dessas desordens, facilitando a aquisição de habilidades motoras.

Durante o desenvolvimento motor típico, para que um padráo de marcha maduro seja adquirido, ocorrem mudanças na coordenação entre os membros e nos membros (SUTHERLAND et al., 1980). A idade em que ocorre a maturação da marcha é um dado controverso, considera-se que ela ocorre em torno de quatro anos (SUTHERLAND, 1997), sete anos (BERKER, YALÇIN et al., 2008; QUINBY; ABRAHAM, 2005), a partir dos sete anos (HOLM et al., 2009) ou, ainda, acredita-se que aos 9,7 anos de idade ainda está em processo de maturação (MORAIS FILHO; REIS; KAWAMURA, 2010).

De acordo com Sutherland et al. (1980, p. 353), com o processo de maturação da marcha

[...] ocorre aumento na velocidade da marcha, aumento do comprimento do passo e diminuição da base de suporte (SUTHERLAND et al., 1980, p. 353).

Nas crianças com PC, uma das consequências decorrentes de limitaçóes motoras mais frequente é o déficit na capacidade de deambulação. A aquisição da marcha por essas crianças apresenta-se como um desafio devido a dificuldades em manterem o equilíbrio em situaçóes estáticas, bem como quando transitam em diferentes sequências de controle dinâmico (STACKHOUSE et al., 2007). A variação na magnitude de parâmetros relacionados à marcha, entre outros fatores, pode estar relacionada ao desenvolvimento motor, patologias e desequilíbrios posturais (MANN et al., 2008).

Para aquisição de uma marcha funcional e independente, muitas vezes é necessário um dispositivo de apoio. Existem vários dispositivos auxiliares de locomoçáo (andadores, muletas e bengalas) que podem fornecer diferentes graus de mobilidade a essas crianças (BERKER; YALÇIN, 2008).

Na prática clínica, o treino de marcha é um recurso frequentemente utilizado com a finalidade de possibilitar maior independência funcional. A partir disso, este estudo tem como objetivo avaliar características cinemáticas da marcha de uma criança com diplegia espástica durante a deambulaçáo auxiliada por diferentes modalidades de apoio (apoio de uma muleta, duas muletas e cintura escapular) e assim determinar a forma mais adequada de treinar a deambulação com base nas características biomecânicas da marcha.

\section{Materiais e métodos}

A presente pesquisa caracteriza-se como descritiva do tipo estudo de caso. Participou do estudo uma criança do sexo feminino, com diagnóstico de PC do tipo diplegia espástica, nove anos de idade, massa corporal de $37,8 \mathrm{~kg}$ e 1,45 m de estatura. A criança avaliada foi classificada no nível III do Gross Motor Function Classification System - GMFCS (ROSENBAUM et al., 2002), uma vez que era capaz de deambular em espaços internos e externos usando um par de muletas canadenses como aparelho auxiliar de locomoção.

Essa criança nasceu de parto prematuro de 32 semanas e permaneceu na incubadora e na ventilação mecânica por 15 dias. Frequenta sessões de 50 minutos de atendimento fisioterapêutico, três vezes por semana, desde os seis meses de idade. Além disso, realiza em casa dez minutos diários de exercícios indicados pelo fisioterapeuta. Realizou tenotomia bilateral de flexores de quadril, joelho, tornozelo e pé esquerdo aos sete anos de idade. Recebeu $50 \mathrm{U}$ de toxina botulínica tipo A no músculo isquiotibial medial direito sete meses antes da avaliação. Iniciou marcha com muletas canadenses três meses antes da avaliação, sendo essa realizada anteriormente com o uso de andador.

A coleta de dados aconteceu no laboratório de biomecânica da Universidade Estadual de Santa Catarina. Após receber orientaçóes sobre todos os procedimentos aos quais seria submetida, a criança foi familiarizada com o local e as atividades a serem executadas durante a coleta de dados. O responsável pela criança foi convidado a ler e assinar o Termo de Consentimento Livre e Esclarecido e o consentimento para filmagens aprovado pelo Comitê de Ética da instituição (protocolo n' 263/2009). 
Obtidos os dados de identificação, a criança, acompanhada pelo seu responsável, foi submetida a uma anamnese e solicitada a colocar uma vestimenta adequada aos procedimentos de coleta de dados, de maneira que os marcadores reflexivos utilizados durante a avaliação ficassem expostos. Em seguida foram realizadas as medidas antropométricas e as avaliaçóes clínicas do grau de espasticidade e da função motora ampla. As variáveis obtidas a partir das avaliações clínicas foram: grau de espasticidade, função motora ampla e habilidades funcionais.

A massa corporal e estatura foram obtidas por meio de uma balança digital da marca Filizola com resolução de $100 \mathrm{~g}$ e um estadiômetro montado sobre uma parede do laboratório e utilizado em conjunto com um esquadro mantido em ângulo reto, com escala de medida de $0,1 \mathrm{~cm}$. O grau de espasticidade dos membros superiores e inferiores foi verificado por meio da Escala Modificada de Ashworth (BOHANNON; SMITH, 1987). A função motora ampla foi avaliada através do Gross Motor Function Measure - GMFM-88 (RUSSELL et al., 2002). Esse teste apresenta 88 itens que são mensurados pela observação das crianças e classificados em uma escala ordinal de quatro pontos. Os itens são agrupados em cinco dimensões: deitar e rolar (A); sentar (B); engatinhar e ajoelhar (C); em pé (D); e andar, correr e pular (E). Neste estudo optamos por utilizar somente as dimensóes $\mathrm{D}$ e $\mathrm{E}$, pois essas estáo diretamente relacionadas com a marcha que é o foco principal deste estudo.

Para a aquisição dos dados cinemáticos foram fixados marcadores reflexivos circulares de $1 \mathrm{~cm}$ de diâmetro no hemicorpo direito da criança, nos seguintes pontos anatômicos: maléolo lateral, linha articular do joelho, trocânter maior do fêmur e acrômio da escápula. Logo após, a criança foi posicionada na pista de caminhada e solicitada a caminhar em uma velocidade confortável com os braços soltos ao longo do corpo por pelo menos $6 \mathrm{~m}$ em uma direção. Para limitar os efeitos de sequência, a avaliação entre as diferentes modalidades de apoio foi determinada através de sorteio. Foram realizadas cinco repetiçóes da marcha com cada modalidade de apoio, na seguinte ordem: duas muletas, uma muleta e apoio na cintura escapular (Figura 1).

Para obtenção das variáveis cinemáticas no plano sagital foi utilizado o sistema de aquisição de imagens Peak Motus (Peak Performance Technologies Inc., Englewood, EUA), com uma câmera de vídeo com taxa de aquisiçáo de $60 \mathrm{~Hz}$, fixada sobre um tripé localizado perpendicularmente ao plano de movimento e com um holofote posicionado sobre a câmera, para a luz refletir nos marcadores. Para calibração do sistema foi utilizado um calibrador com quatro pontos formando um quadrado com as dimensões 1,2 $\mathrm{m} \times 1,2 \mathrm{~m}$. O software Ariel Performance Analysis System (APAS) foi utilizado para digitalização das imagens, que foi realizada manualmente, quadro a quadro. Os dados cinemáticos, após a digitalização no software Ariel Performance Analysis System (APAS), foram filtrados com filtro de passa-baixa com frequência de corte de $6 \mathrm{~Hz}$.

Foram analisados os ângulos de flexão do joelho e do quadril no plano sagital direito e obtidas as seguintes variáveis angulares (graus de movimento): flexão de quadril no contato inicial; máxima flexão de quadril na fase de balanço; máxima extensão de quadril; flexão de joelho no contato inicial; máxima

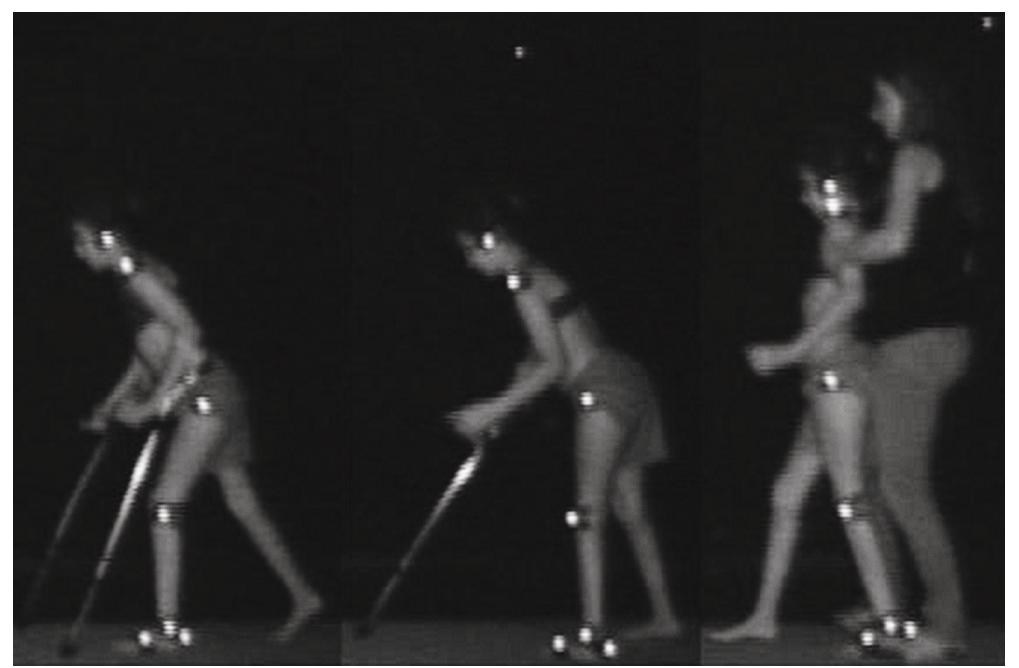

Figura 1. Criança durante o procedimento de aquisição dos dados cinemáticos com o apoio em duas muletas, uma muleta e na cintura escapular. 
extensáo de joelho na fase de apoio; e pico de flexáo do joelho na fase de balanço. As variáveis espaçotemporais obtidas (valores absolutos e normalizados) foram: tempo da passada (s); comprimento da passada $(\mathrm{m})$; velocidade da passada $(\mathrm{m} / \mathrm{s})$; tempo de apoio (\% do ciclo); tempo de balanço (\% do ciclo); comprimento da passada (\% da estatura); e velocidade da passada ( $\mathrm{m} / \mathrm{s} /$ estatura).

\section{Resultados}

Em relação ao grau de espasticidade segundo a Escala Modificada de Ashworth, foi observado que a criança avaliada apresentou no membro superior direito o grau 0 (tônus muscular normal), no membro superior esquerdo o grau 1 (hipertonia leve, aumento do tônus muscular, mínima resistência no final do seu arco de movimento), no membro inferior direito e no esquerdo o grau 1+ (hipertonia leve, aumento do tônus muscular, mínima resistência em menos da metade de seu arco de movimento). Em relação à função motora ampla (GMFM), na dimensão D (em pé), a criança avaliada pontuou 16 de 39 pontos, o que corresponde a 41,02\% dos pontos possíveis. $\mathrm{Na}$ dimensão $\mathrm{E}$ (andar, correr e pular), pontou 15 de 72 possíveis, o que equivale a $20,83 \%$. A soma dividida pelo número de dimensóes avaliadas corresponde ao escore total do GMFM, que resultou em 30,92\%.

Os resultados dos parâmetros espaço-temporais e angulares da marcha durante o apoio em duas muletas, uma muleta e na cintura escapular são apresentados nas Tabelas 1 e 2, respectivamente.

\section{Discussão}

Crianças classificadas nos níveis superiores do GMFCS indicam maior comprometimento motor e apresentam menor funcionalidade. Segundo a avaliação clínica da funçáo motora pelo GMFM-88, o ponto de corte médio de $40 \%$ representa crianças com menor capacidade nas habilidades motoras amplas, correspondendo aproximadamente à capacidade média de crianças com nível de funcionalidade IV pelo GMFCS (AVERY et al., 2003, p. 702).

No presente estudo, a criança avaliada encontra-se no nível III pelo GMFCS e no GMFM-88 teve

Tabela 1. Valores médios e desvio-padrão dos parâmetros espaço-temporais da marcha de uma criança diplégica durante o apoio em duas muletas, uma muleta e na cintura escapular.

\begin{tabular}{lccc}
\hline & Duas muletas & Uma muleta & Cintura escapular \\
\hline Valores absolutos & & & \\
Tempo da passada (s) & $1,64 \pm 0,20$ & $1,82 \pm 0,26$ & $1,89 \pm 0,13$ \\
Comprimento da passada (m) & $0,66 \pm 0,06$ & $0,32 \pm 0,08$ & $0,68 \pm 0,03$ \\
$\quad$ Velocidade da passada (m/s) & $0,41 \pm 0,08$ & $0,18 \pm 0,05$ & $0,36 \pm 0,04$ \\
Valores normalizados & & & \\
Tempo de apoio (\% ciclo) & $68,49 \pm 4,93$ & $77,28 \pm 7,48$ & $69,33 \pm 2,36$ \\
Tempo de balanço (\% ciclo) & $31,51 \pm 4,93$ & $22,72 \pm 7,48$ & $30,67 \pm 2,36$ \\
Comprimento da passada (\% estatura) & $45,32 \pm 4,33$ & $22,03 \pm 5,59$ & $47,27 \pm 2,43$ \\
Velocidade da passada (m/s/estatura) & $0,28 \pm 0,05$ & $0,13 \pm 0,03$ & $0,25 \pm 0,03$ \\
\hline
\end{tabular}

Tabela 2. Valores médios e desvio-padrão dos parâmetros angulares da marcha de uma criança diplégica no plano sagital durante o apoio em duas muletas, uma muleta e na cintura escapular.

\begin{tabular}{llll}
\hline & \multicolumn{1}{c}{ Duas muletas } & Uma muleta & Cintura escapular \\
\hline Quadril & & & \\
$\quad$ Flexão no contato inicial & $47,99 \pm 4,42$ & $43,21 \pm 2,95$ & $5,23 \pm 4,06$ \\
$\quad$ Máxima flexão na fase de balanço & $56,83 \pm 4,33$ & $53,35 \pm 7,12$ & $21,28 \pm 2,98$ \\
$\quad$ Máxima extensão & $31,85 \pm 2,80$ & $33,03 \pm 8,65$ & $-6,42^{*} \pm 6,28$ \\
$\quad$ Joelho & $22,68 \pm 6,12$ & $13,53 \pm 3,46$ & $10,57 \pm 0,90$ \\
$\quad$ Flexão no contato inicial & $6,52 \pm 2,38$ & $7,45 \pm 2,31$ & $6,63 \pm 1,88$ \\
$\quad$ Máxima extensão na fase de apoio & $47,56 \pm 3,89$ & $34,73 \pm 10,18$ & $52,76 \pm 4,61$ \\
$\quad$ Pico de flexão na fase de balanço & & & \\
Tronco & $35,38 \pm 2,61$ & $33,85 \pm 4,70$ & $-2,80^{* *} \pm 0,81$ \\
$\quad$ Inclinação do tronco &
\end{tabular}

*O valor negativo corresponde a uma hiperextensão de quadril; **O valor negativo corresponde a uma anteriorização do quadril associada a uma extensão de tronco. 
como média 30,92\% e 20,83\% nas dimensóes D e $\mathrm{E}$, respectivamente, apresentando uma pontuaçáo abaixo do mínimo a ser considerado para crianças com maior comprometimento funcional. Apesar de ter sido observado que a espasticidade foi leve, a grave limitação funcional indica necessidade de aparelhos auxiliares para a locomoçáo.

A marcha de crianças com PC, quando comparada à de crianças com desenvolvimento típico, apresenta diferentes parâmetros espaço-temporais (JERÔNIMO et al., 2007). Holm et al. (2009), aferindo crianças com desenvolvimento típico e com idade entre sete e 12 anos, encontraram como média de velocidade de marcha $1,5 \mathrm{~m} / \mathrm{s}$. A criança avaliada no presente estudo apresentou como menor valor de marcha, com o apoio de uma muleta, $0,18 \mathrm{~m} / \mathrm{s}$, e como maior valor, com apoio de duas muletas, 0,41 $\mathrm{m} / \mathrm{s}$. Outros estudos encontraram maior velocidade da passada 0,62 m/s (DINI; DAVID, 2009), 0,64 $\mathrm{m} / \mathrm{s}$ e $0,71 \mathrm{~m} / \mathrm{s}$ (FRANCO, 2009), mas esses valores correspondem a crianças com PC com menor comprometimento motor, classificadas no GMFCS no nível I e II. A velocidade reduzida é proporcional ao comprometimento funcional (DAMIANO; ABEL, 1998). As limitaçôes motoras das crianças com PC podem contribuir para a diminuição da velocidade da marcha (DINI; DAVID, 2009). A maior velocidade da passada ocorreu com apoio de duas muletas, caracterizando-se como o mais indicado nesse parâmetro.

Com relação ao comprimento da passada, ele está diretamente relacionado com o aumento da idade, principalmente nos primeiros quatro anos (DUSING; THORPE, 2007). No estudo de Franco (2009), os valores médios encontrados foram $0,75 \mathrm{~m}$ e $0,67 \mathrm{~m}$ para crianças com diplegia, respectivamente, no nível I e II no GMFCS (FRANCO, 2009). Na avaliação realizada, a criança era mais comprometida, mas foi observado que tanto na marcha com apoio escapular quanto na com apoio em duas muletas os valores encontrados estão próximos aos valores encontrados pelos autores acima citados. No entanto, a partir dos resultados observados, o apoio com uma muleta mostrou-se menos indicado em relaçáo à variável comprimento da passada.

Durante a marcha adulta, cerca de $62 \%$ do ciclo ocorre durante a fase de apoio, enquanto $38 \%$ ocorre durante a fase de balanço (SUTHERLAND, 1997). Já na marcha de crianças de sete anos a duração da fase de apoio é de aproximadamente $38 \%$ e o comprimento do passo e a velocidade não alcançam os mesmos valores dos adultos até que a criança atinja seu crescimento (KEEN, 1993). Neste estudo pôde-se notar que a marcha com duas muletas apresentou menor tempo de apoio $(68,49 \%)$, seguida pela marcha com apoio escapular $(69,33 \%)$ e pela com o apoio com uma muleta $(77,28 \%)$. Os valores encontrados para a fase de apoio e, consequentemente, para a fase de balanço estáo mais próximos do valor supracitado durante a marcha com apoio de duas muletas.

Em relação às variáveis angulares, 22 crianças com desenvolvimento típico na faixa etária de seis a 10 anos de idade apresentaram o ângulo de flexão do quadril de $22^{\circ}$ no contato inicial (DAVID; ÁVILA, 2004). No presente estudo, os valores encontrados nas três formas de apoio difere do estudo supracitado, sendo que o mais alterado é durante o apoio com duas muletas. Já para a máxima flexão de quadril durante a fase de balanço e para a máxima extensão de quadril de crianças típicas foram encontrados, respectivamente, valores de $25^{\circ}$ e $20^{\circ}$ (DAVID; ÁVILA, 2004). Comparando-se esses com os do presente estudo, os valores da máxima flexão de quadril durante a fase de balanço na marcha com apoio escapular $\left(21,28^{\circ}\right)$ e os valores da máxima extensão de quadril durante a marcha com duas muletas $\left(31,85^{\circ}\right)$ se apresentaram mais próximos dos valores citados anteriormente.

David e Ávila (2004) observaram que crianças com desenvolvimento típico apresentam a flexão do joelho durante o contato inicial com média de $0^{\circ}$. De acordo com esse valor, a marcha com apoio escapular apresentou a média mais próxima nesse parâmetro. Crianças com PC geralmente apresentam redução na extensão de joelho, o que ocasiona diminuição no comprimento do passo e redução na velocidade durante a marcha, implicando em limitaçóes no desempenho funcional (VAN DER KROGT et al., 2009).

No presente estudo, nas três diferentes formas de apoio da marcha, a máxima extensão de joelho na fase de apoio apresentou valores próximos aos obtidos por David e Ávila (2004) com crianças com desenvolvimento típico. Apesar da máxima extensão de joelho na fase de apoio não estar alterada e do pico de flexão de joelho nos apoios de duas muletas e na cintura escapular serem próximos dos valores obtidos por David e Ávila (2004), verificou-se que a velocidade da marcha estava reduzida, o que pode ser justificado pela espasticidade extensora em membros inferiores, comum nessas crianças (NORKIN, 1993).

Em relação ao pico de flexão de joelho na fase de balanço, somente as médias angulares obtidas durante o apoio nas duas muletas e na cintura escapular foram próximas dos valores de crianças típicas (DAVID; ÁVILA, 2004). Uma velocidade adequada de flexão do joelho durante a fase de pré-balanço é importante na adequada flexão de joelho na fase de balanço, dessa maneira, uma menor velocidade de flexão de joelho no pré-balanço pode colaborar para a marcha com joelho rígido e, em consequência, 
para a diminuição da flexão do joelho na fase de balanço (GOLDBERG et al., 2004). Damiano et al. (2006), comparando crianças com PC e crianças com desenvolvimento motor típico, notaram que as crianças com PC apresentaram um menor pico de flexão, além da reduzida velocidade da marcha.

A falha no controle motor seletivo de flexão de quadril em determinadas fases da marcha, como pré-balanço e balanço inicial, leva à atuação do músculo reto femoral para compensar a flexão inadequada de quadril. Essa, por sua vez, se opóe à flexão do joelho e à ação extensora do quadril, resultando em um menor comprimento do passo (PERRY, 2005). Apesar de os valores de flexão e extensão de quadril se apresentarem alterados, eles não influenciaram a flexão do joelho e o comprimento da passada, pois esses parâmetros estavam dentro da normalidade. Contudo, havendo alteração na extensáo do quadril pode haver comprometimento da estabilidade do sujeito (PERRY, 2005).

$\mathrm{Na}$ marcha atípica, a pélvis e o tronco podem ser usados para compensar os déficits de membros inferiores (LEROUX; FUNG; BARBEAU, 2002). Conforme Norkin (1993), a perda de controle sobre a atividade muscular pode resultar, entre outras alteraçôes da marcha, em inclinação do tronco para a frente ou para trás. No presente estudo, o apoio em duas muletas ocasionou maior inclinação do tronco, o que poderia ser considerado maior compensação durante a utilização desse apoio. Contudo, com duas muletas a criança apresentou valores mais próximos do considerado normal nos parâmetros velocidade da passada, tempo de apoio e máxima extensão de quadril. $\mathrm{O}$ apoio na cintura escapular ocasionou menor inclinaçáo do tronco, mas os parâmetros estavam melhores na máxima flexão de quadril na fase de balanço e flexão do joelho no contato inicial. Já com apoio em uma muleta observou-se que o único parâmetro próximo ao estudo de David e Ávila (2004) foi a máxima extensão do joelho na fase de apoio.

O desenvolvimento da marcha é uma atividade motora essencial para a criança. Com base nas características espaço-temporais e angulares entre as três diferentes modalidades de apoio avaliadas, o apoio em duas muletas e na cintura escapular apresentaram um comportamento bastante similar. Contudo o objetivo do tratamento fisioterapêutico é promover maior funcionalidade e independência nas atividades de vida diária, dessa forma a marcha realizada com apoio em duas muletas foi considerada a mais indicada.

\section{Conclusão}

De acordo com os resultados encontrados neste estudo, existe variabilidade tanto nos parâmetros espaço-temporais quanto nos parâmetros angulares da marcha entre as diferentes modalidades de apoio. Verificou-se que a marcha com duas muletas seria a modalidade de apoio mais indicada, considerando-se a funcionalidade e a independência da criança. Contudo, a marcha com apoio na cintura escapular pode ser indicada para o treinamento de marcha durante a prática clínica por estimular diferentes parâmetros espaço-temporais e angulares. A coleta de dados foi realizada com uma criança e, dessa forma, a pouca possibilidade de generalizaçóes de um estudo de caso apresenta limitaçóes inferenciais. Existe necessidade de mais estudos, com uma amostra maior, visando estabelecer a melhor forma de treinamento da marcha para crianças com PC do tipo diplegia espástica.

\section{Referências}

AVERY, L. M. et al. Rasch Analysis of the Gross Motor Function Measure: Validating the Assumptions of the Rasch model to Create an Interval-Level Measure. Archives Physical Medicine and Rehabilitation, Reston, v. 84, p. 697-705, 2003. http://dx.doi.org/10.1016/ S0003-9993(02)04896-7

BERKER, A. N.; YALCIN, M. S. Cerebral Palsy: Orthopedic Aspects and Rehabilitation. Pediatric Clinics of North America, Philadelphia, v. 55, n. 5, p. 1209-25, 2008. PMid:18929061. http://dx.doi.org/10.1016/j. pcl.2008.07.011

BOHANNON, R. W.; SMITH, M. B. Interrater reliability of a modified Ashworth scale of muscle spasticity. Physical Therapy, NewYork, v. 67, p. 206-207, 1987.

DAMIANO, D. L.; ABEL, M. F. Functional outcomes of strength training in spastic cerebral palsy. Archives Physical Medicine and Rehabilitation, Reston, v. 79, p. 119-25, 1998. PMid:16311188. http://dx.doi.org/10.1016/j. gaitpost.2004.10.007

DAMIANO, D. et al. Relationship of spasticity to knee angular velocity and motion during gait in cerebral palsy. Gait \& Posture, Amsterdam, v. 23, p. 1-8, 2006.

DAVID, A. C.; ÁVILA, A. O. V. Análise cinemática dos membros inferiores durante o andar em crianças. Brazilian Journal of Biomechanics, São Paulo, v. 5, n. 8, p. 25-32, 2004.

DINI, P. D.; DAVID, A. C. Repetibilidade dos parâmetros espaço-temporais da marcha: comparaçáo entre crianças normais e com paralisia cerebral do tipo hemiplegia espástica. Revista Brasileira de Fisioterapia, São Carlos, v. 13, n. 3, p. 215-222, 2009. http://dx.doi.org/10.1590/ S1413-35552009005000031 
DUSING, S. C.; THORPE, D. E. A normative sample of temporal and spatial gait parameters in children using the Gaitritel electronic walkway. Gait \& Posture, Amsterdam v. 25, p. 135-139, 2007. PMid:16875823. http://dx.doi. org/10.1016/j.gaitpost.2006.06.003

FRANCO, R. L. L. Análise cinemática da marcha de crianças diplégicas nivel I e II do GMFCS. 2009. $66 \mathrm{f}$. Dissertação (Mestrado em Ciências da Reabilitação)Universidade Federal de Minas Gerais, Belo Horizonte, 2009.

GOLDBERG, S. R. et al. Muscles that influence knee flexion velocity in double support: implications for stiff-knee gait. Journal of Biomechanics, Philadelphia, v. 37, p. 1189-1196, 2004. PMid:15212924. http://dx.doi. org/10.1016/j.jbiomech.2003.12.005

HOLM, I. et al. A normative sample of gait and hopping on one leg parameters in children 7-12 years of age. Gait \& Posture, Amsterdam, v. 29, p. 317-321, 2009. PMid:19019681. http://dx.doi.org/10.1016/j. gaitpost.2008.09.016

JERÔNIMO, B. P. et al. Variáveis espaço-temporais da marcha de crianças com paralisia cerebral submetidas a eletroestimulação no músculo tibial anterior. Revista Brasileira de Fisioterapia, São Carlos, v. 11, n. 4, p. 261-66, 2007. http://dx.doi.org/10.1590/ S1413-35552007000400004

KEEN, M. Early Development and Attainment of Normal Mature Gait. Journal of Prosthetics \& Orthotics, Washington, v. 5, n. 2, p. 35-38, 1993.

LEROUX, A.; FUNG, J.; BARBEAU, H. Postural adaptation to walking on inclined surfaces: I. Normal strategies. Gait \& Posture, Amsterdam, v. 15, n. 1, p. 64-74, 2002. http://dx.doi.org/10.1016/S0966-6362(01)00181-3

MANN, L. et al. A marcha humana: investigação com diferentes faixas etárias e patologias. Motriz, Rio Claro, v. 14, n. 3, p. 346-53, 2008.

MORAIS FILHO, M. C.; REIS, R. A.; KAWAMURA, C. M.; Avaliação do padrão de movimento dos joelhos e tornozelos durante a maturação da marcha normal. Acta Ortopédica Brasileira, São Paulo, v. 18, n. 1, p. 23-25, 2010.

NORKIN, C. Análise da Marcha. In: O’SULLIVAN S. B.; SCHMITZ T. J. Fisioterapia: Avaliação e Tratamento. São Paulo: Manole, 1993. p. 225-249.

PERRY, J. Análise de Marcha-marcha normal. Barueri: Manole, 2005.

QUINBY, J. M.; ABRAHAM, A. Musculoskeletal problems in cerebral palsy. Current Paediatrics, Melbourn, v. 15, p. 9-14, 2005.

RIES, L. G. K.; BÉRZIN, F. Sinais e Sintomas de Disfunção Temporomandibular em crianças portadoras de Paralisia Cerebral. Revista Brasileira de Fisioterapia, São Carlos, v. 9, n. 3, p. 341-346, 2005.

ROSENBAUM, P. L. et al. Prognosis for Gross Motor Function in Cerebral Palsy: Creation of Motor Development Curves. Journal of the American the Medical Association, Chicago, v. 288, p. 1357-1363, 2002. http:// dx.doi.org/10.1001/jama.288.11.1357

RUSSELL, D. et al. Gross Motor Function Measure (GMFM-66 \& GMFM-88): user's manual. Canadá: McMaster University, CanChild Centre for Childhood Disability Research, 2002.

STACKHOUSE, C. et al. Gait initiation in children with cerebral palsy. Gait \& Posture, Amsterdam, v. 26, p. 301-308, 2007. PMid:17081756. http://dx.doi. org/10.1016/j.gaitpost.2006.09.076

SUTHERLAND, D. The development of mature gait. Gait \& Posture, Amsterdam, v. 6, p. 163-170, 1997. http:// dx.doi.org/10.1016/S0966-6362(97)00029-5

SUTHERLAND, D. H. et al. The Development of Mature Gait. Journal of Bone and Joint Surgery, Needhan, v. 62, n. 3, p. 336-353, 1980.

VAN DER KROGT, M. M. et al. Walking speed modifies spasticity effects in gastrocnemius and soleusin cerebral palsy gait. Clinical Biomechanics, Oxford, v. 24, p. 422-428, 2009. PMid:19349103. http://dx.doi.org/10.1016/j. clinbiomech.2009.02.006

\section{Contribuição dos Autores}

Todas as autoras colaboraram em todas as etapas da elaboração do texto.

\section{Notas}

${ }^{1}$ Foram respeitados os princípios éticos de acordo com a resolução vigente. O estudo foi enviado ao Comitê de Ética em Pesquisa em Seres Humanos da Universidade do Estado de Santa Catarina (UDESC) e aprovado sob protocolo número 263/2009. 